\title{
The off-axis jet structure in Mrk 501 at mm-wavelengths
}

\author{
Shoko Koyama ${ }^{* a}$, Motoki Kino ${ }^{b}$, Marcello Giroletti $^{c}$, Akihiro Doi $^{d}$, Hiroshi Nagai ${ }^{e}$, \\ Kazuhiro Hada ${ }^{c, e}$, Kotaro Niinuma ${ }^{f}$, Monica Orienti ${ }^{c}$, Gabriele Giovannini ${ }^{g, c}$, \\ Eduardo Ros $^{a, h, i}$, Tuomas Savolainen ${ }^{j, a}$, Miguel. Á. Pérez-Torres ${ }^{k, l}$, and Thomas P. \\ Krichbaum $^{a}$ \\ ${ }^{a}$ Max-Planck-Institute für Radioastronomie, Auf dem Hügel 69, D-53121 Bonn, Germany \\ ${ }^{b}$ Korea Astronomy and Space Science Institute, 776 Daedeokdae-ro, Yuseong-gu, Daejeon, \\ 305-348, Republic of Korea \\ ${ }^{c}$ INAF Istituto di Radioastronomia, via Gobetti 101, I-40129 Bologna, Italy \\ ${ }^{d}$ Institute of Space and Astronautical Science, Japan Aerospace Exploration Agency, 3-1-1 \\ Yoshinodai, Chuou-ku, Sagamihara, Kanagawa 252-5210, Japan \\ ${ }^{e}$ National Astronomical Observatory of Japan, 2-21-1 Osawa, Mitaka, Tokyo 181-8588, Japan \\ ${ }^{f}$ Graduate School of Science and Engineering, Yamaguchi University, Yamaguchi 753-8511, \\ Japan \\ ${ }^{g}$ Dipartimento di Astronomia, Università di Bologna, via Ranzani 1, I-40127 Bologna, Italy \\ ${ }^{h}$ Observatori Astronòmic, Universitat de València, E-46980 Paterna, València, Spain \\ ${ }^{i}$ Departament d'Astronomia i Astrofísica, Universitat de València, E-46100 Burjassot, València, \\ Spain \\ ${ }^{j}$ Aalto University Metsähovi Radio Observatory, FIN-02540 Kylmälä, Finland \\ ${ }^{k}$ Instituto de Astrofísica de Andalucía, Glorieta de las Astronomía, s/n, E-18008 Granada, Spain \\ ${ }^{l}$ Centro de Estudios de la Física del Cosmos de Aragón, E-44001 Teruel, Spain \\ E-mail: Skoyamadmpifr-bonn.mpq.de
}

\begin{abstract}
We present results from $43 \mathrm{GHz}$ (VLBA, six epochs from 2012.2 to 2013.2) and $86 \mathrm{GHz}$ (GMVA, one epoch in 2012.4) observations toward the basis of the jet in the TeV Blazar Mrk 501. The 43-GHz data analysis reveals a new feature located northeast of the radio core, with a flux density of several tens of mJy, perpendicularly to the jet axis. The $86-\mathrm{GHz}$ image shows the jet feature located 0.75 mas southeast of the radio core, which is consistent with previous result. The location of Gaussian model for 0.75 mas feature does not coincide with those for the jets in the $43-\mathrm{GHz}$ image, however, a distribution of emission is found. We also discuss the spectral indices of the core, the northeast feature, and the jet feature between $43 \mathrm{GHz}$ and $86 \mathrm{GHz}$, which show flat-tosteep, steep, and flat-to-invert, respectively.
\end{abstract}

12th European VLBI Network Symposium and Users Meeting,

7-10 October 2014

Cagliari, Italy

\footnotetext{
* Speaker.
} 


\section{Introduction}

The innermost structure of relativistic jets in active galactic nuclei are considered to be related to the properties of the regions where the jets are formed, collimated, and accelerated. Ultra-high resolution Very Long Baseline Interferometry (VLBI) observations are the best tools to explore the parsec scale structure of jets. Recently, the Event Horizon Telescope at $230 \mathrm{GHz}(1.3 \mathrm{~mm})$ has revealed the off-axis jet structure within 0.3 mas from the core in two quasars [1, 2]. However, in BL Lac Objects, such off-axis jet structure have not been studied.

The TeV blazar Mrk 501 is one of the best BL Lac Objects to resolve the innermost jet because of its proximity $(z=0.034,1$ mas $=0.66 \mathrm{pc})$ and brightness. The VLBI images show different jet position angles at different scales [3]. The previous Very Long Baseline Array (VLBA) $43 \mathrm{GHz}$ and Global mm-VLBI Array (GMVA) $86 \mathrm{GHz}$ images show clear limb-bright structure [4, 5], while they do not show clear off-axis jet structure at the innermost part.

\section{Observations and Data Reduction}

We observed this target in six different epochs by the VLBA at $43 \mathrm{GHz}$ and one epoch by the GMVA at $86 \mathrm{GHz}$, with $8 \mathrm{IFs}$ of $16 \mathrm{MHz}$ bandwidth each. The total on-source time for Mrk 501 was approximately one hour for the $43 \mathrm{GHz}$ data, and four hours for the $86 \mathrm{GHz}$ data. Phase and delay offset between different sub-bands were solved by using the calibrator 3C 345 . For the $43 \mathrm{GHz}$ data, after the standard initial calibrations in AIPS, the final images were produced after iterations of CLEAN, phase and amplitude self-calibration processes in difmap. For the $86 \mathrm{GHz}$ data, fringe fitting on Mrk 501 were performed with a small search window of a few tens of nsec and $\mathrm{mHz}$ in delay and rate respectively, and a low SNR threshold, after applying the delay and rate solutions of 3C 345. Fringe solutions of Mrk 501 were not derived among transatlantic baselines, however, weak coherent phases were found. Gaussian model fitting and phase self-calibration were done in difmap, without amplitude self-calibration due to low SNR of the visibilities.

\section{Results}

In Figure $\mathbb{W}$, we find a new component (labeled NE) located almost perpendicularly to the main jet axis (labeled C1, C2, C3). The location (angular separation, position angle, and flux) of NE changes randomly. We also confirmed the existence of NE using the slice profiles of the CLEAN images across the center of Gaussian models for core and NE. There is a clear emission

at the northeast of the core for all epochs with the peak flux of $36 \mathrm{mJy}^{\text {beam }}{ }^{-1}$, the position of 0.18 mas from the core, and the FWHM of 0.24 mas in average. These results suggest that the limb-brightened structure of Mrk 501 apparently stars in the nearest vicinity of the core due to the small viewing angle.

Figure 2 -Left shows the total-intensity image obtained by the GMVA at $86 \mathrm{GHz}$ at a resolution of $205 \mu$ as $\times 37 \mu$ as $\left(P A-5.11^{\circ}\right)$ with fitted Gaussian models. We find a tentative jet knot located at $(r, \theta)=\left(0.75\right.$ mas, $\left.159^{\circ}\right)$ with a SNR of $\sim 5 \sigma$. This knot could correspond to one detected by the GMVA at $86 \mathrm{GHz}$ in [4], located at $(r, \theta)=\left(0.73\right.$ mas, $\left.172^{\circ}\right)$. The flux of the core is $197 \pm 59 \mathrm{mJy}$ and that of the tentative jet knot is $19 \pm 8 \mathrm{mJy}$. 

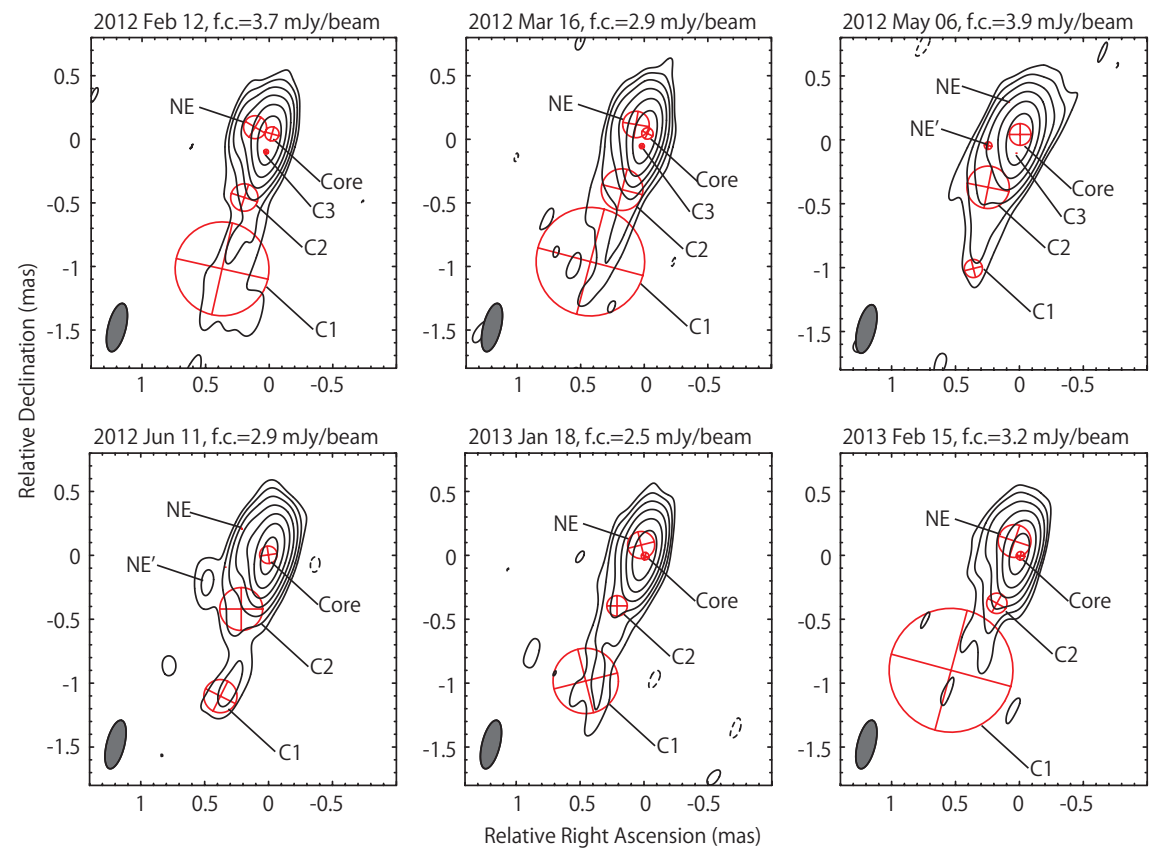

Figure 1: $43 \mathrm{GHz}$ uniform weighted VLBA images with fitted circular Gaussian components. The restoring beam is 0.39 mas $\times 0.14$ mas in $P A-14^{\circ}$, plotted in the bottom-left corner. Observing date and the first contour (f.c.) are plotted above each map. The first contour are all set to three times the rms noise level of each map, increasing by a factor of 2 .

Since the 86-GHz data and the third-epoch VLBA data at $43 \mathrm{GHz}$ were obtained within two weeks, we compare these two images. In the $43-\mathrm{GHz}$ image, there are no Gaussian models at $\sim 0.75$ mas southeast of the core, however, a distribution of emission is found. The spectral index of the integrated core fluxes over Gaussian models between $43 \mathrm{GHz}$ and $86 \mathrm{GHz}$ is $\alpha=-0.8 \pm 0.5$, where we define the flux density at observing frequency $v$ as $S_{v} \propto v^{\alpha}$. This flat-to-steep spectral tendency is consistent with the power law index of the core reported in [4] ( $\alpha \sim-0.5$ above $8 \mathrm{GHz})$. Using the model-fit flux of the $43-\mathrm{GHz}$ image and the $3 \sigma$ upper limit in the $86-\mathrm{GHz}$ image, the spectral index of NE is estimated to be $\alpha \leq-0.8$, which is steeper than that for the radio core. We also estimate the spectral index at the southeast jet as $\alpha=-0.1 \pm 0.5$ by integrating the flux within the same region between the two images using the AIPS task IMSTAT. Due to the large spectral index error, this is not a stringent result. However, despite of the steep spectrum of the core, the southeast jet spectrum is flat-to-inverted and it is inconsistent with one at lower frequencies [e.g., 6]. This tendency is also seen in the spectral index map shown in Figure 2 -Right.

\section{Summary and future prospects}

We have explored the parsec-scale regions in the jet of Mrk 501 using the VLBA at $43 \mathrm{GHz}$ and the GMVA at $86 \mathrm{GHz}$. We newly find the off-axis jet structure, NE, in the $43 \mathrm{GHz}$ image, and detect a 0.75 mas jet feature in the $86 \mathrm{GHz}$ image. The spectrum of the radio core is flat-to-steep and consistent with previous results, while the jet spectrum is flat-to-invert and inconsistent with one at lower frequencies. 

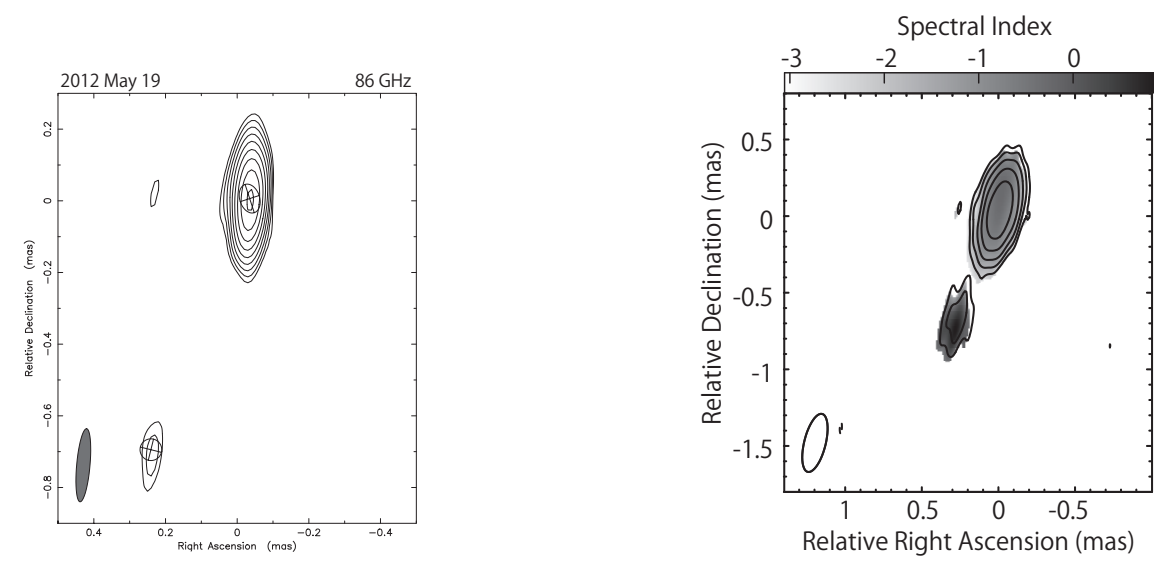

Figure 2: Left: GMVA image of Mrk 501 at $86 \mathrm{GHz}$ with model fit components with the restoring beam of $205 \mu$ as $\times 37 \mu$ as in $P A-5.11^{\circ}$. The peak brightness is $98 \mathrm{mJy}$ beam $^{-1}$, and the contour levels are drawn at $(-1,1,1.141,2, \cdots) \times 5.8 \mathrm{mJy}_{\text {beam }}{ }^{-1}$. The $1 \sigma$ noise level is $1.9 \mathrm{mJy}^{-1}$ beam $^{-1}$. Right: Spectral index map between the GMVA $86 \mathrm{GHz}$ image and the third epoch VLBA $43 \mathrm{GHz}$ image with total intensity contours of restored GMVA image.

This observation cannot confirm the spectral index of the jet feature in Mrk 501 due to the limited $(u, v)$-coverage provided by the array used. To obtain more precise GMVA images, a better $(u, v)$-coverage, achievable by including new stations (e.g., the Korean VLBI Network) in the array, is indeed recommended.

Acknowledgments This paper is partially based on observations carried out with the VLBA, the MPIfR $100 \mathrm{~m}$ Effelsberg Radio Telescope, the IRAM Plateau de Bure Millimetre Interferometer, the IRAM $30 \mathrm{~m}$ Millimeter Telescope, the Onsala $20 \mathrm{~m}$ Radio Telescope, and the Metsähovi $14 \mathrm{~m}$ Radio Telescope. The National Radio Astronomy Observatory is a facility of the National Science Foundation operated under cooperative agreement by Associated Universities, Inc. The GMVA is operated by the MPIfR, IRAM, NRAO, OSO, and MRO. Part of this work was done with the contribution of the Italian Ministry of Foreign Affairs and University and Research for the collaboration project between Italy and Japan.

\section{References}

[1] R.-S. Lu, V. L. Fish, J. Weintroub et al., Resolving the Inner Jet Structure of 1924-292 with the Event Horizon Telescope, ApJ 757 (2012) L14

[2] R.-S. Lu, V. L. Fish, K. Akiyama, et al., Fine-scale Structure of the Quasar 3C 279 Measured with $1.3 \mathrm{~mm}$ Very Long Baseline Interferometry, ApJ 772 (2013) 13

[3] M. Giroletti, G. Giovannini, L. Feretti, et al., Parsec-Scale Properties of Markarian 501, ApJ 600 (2004) 127

[4] M. Giroletti, G. Giovannini, W. D. Cotton, et al., The jet of Markarian 501 from millions of Schwarzschild radii down to a few hundreds, A\&A 488 (2008) 905

[5] B. G. Piner, N. Pant, P. G. Edwards, and K. Wiik, Significant Limb-Brightening in the Inner Parsec of Markarian 501, ApJ 690 (2009) L31

[6] T. Hovatta, M. F. Aller, H. D. Aller, et al., MOJAVE: Monitoring of Jets in Active Galactic Nuclei with VLBA Experiments. XI. Spectral Distributions, AJ 147 (2014) 143 